\title{
PROBLEMS OF VARISTOR QUALITY ASSESSMENT DURING EXPLOITATION
}

\author{
Janusz Smulko ${ }^{1)}$, Marek Olesz ${ }^{2)}$, Lech Hasse ${ }^{1)}$, Leszek Kaczmarek ${ }^{1)}$, Grzegorz Lentka ${ }^{1)}$ \\ 1) Gdansk University of Technology, Faculty of Electronics, Telecommunications and Informatics, Department of Metrology and \\ Optoelectronics, G. Narutowicza 11/12,80-233 Gdansk,Poland(هjsmulko@eti.pg.gda.p1) \\ 2) Gdansk University of Technology, Faculty of Electronics, Telecommunications and Informatics, Department of Electrical and Control \\ Engineering, Gdansk, Poland
}

\begin{abstract}
Varistors are commonly used elements which protect power supply networks against high-voltage surges or lightning. Therefore, quality and endurance of these elements is important to avoid losses when an expensive laboratory equipment would not be protected from random overvoltages. Additionally, excessive leakage currents generate serious costs due to high energy consumption. The paper presents shortly properties of varistors that comprized different $\mathrm{ZnO}$ grain types and can have various quality which changes continuously during exploitation (due to exposition to overheating and overvoltage pulses). Therefore, it is important to monitor varistors during their ageing (causing changes within their microstructures). A few methods of varistor property diagnosis were considered and compared with the methods currently applied in laboratory or industry applications. A new measurement (diagnostic) system that can monitor varistors during ageing and can be widely applied in power networks is presented. The proposed system fulfills requirements of the industrial customers which demand various methods for power line protection. The proposed system can be simply developed into a more advanced wireless diagnostic system of long power supply lines.
\end{abstract}

Keywords: varistors, quality, ageing.

\section{Introduction}

Varistors are ubiquitous elements in power supply networks. They protect devices against lightning or high voltage surges and are now generally installed even in household power networks. These elements can comprise various type $\mathrm{ZnO}$ grains and have a nonlinear current-voltage characteristics which alters during exploitation and can diminish varistor protective properties. Varistors change their electrical characteristic due to environmental conditions and excessive overheating in the presence of voltage surges. However, varistors should protect power networks during a long time (at least ten years) without any maintenance. Thus, it is important to monitor varistors during their long-lasting work to assure that the power-supplied devices are guarded against high overvoltage pulses due to economic and safety reasons.

The quality of varistors can be determined by using various methods. Some of the methods are used in different production stages [1-3], other during exploitation [4,5] or in a laboratory only [6] but still there are no universal methods which could be applied for cheap and widespread varistor quality monitoring in their work. The pros and cons of these methods as well as their further development will be described. This is effected in a new measurement unit for varistor monitoring. The main aim of this paper is to explain why such solution was proposed and how it can be used in industrial applications, in power supply lines, as a cheap and multifunctional unit. The presented proposal was developed thanks to cooperation with industrial companies, by including detailed requirements of the customers which assemble varistors for protecting power lines, where a very different probability of occurrence and 
various intensities of voltage surges and climatic conditions (temperature, humidity) are present.

\section{Varistor structures}

Varistors are produced on the basis of zinc oxide $(\mathrm{ZnO})$ with a homogeneous distribution of various dopants - other metal oxides (e.g.: bismuth, cobalt, manganese) - in the $\mathrm{ZnO}$ mixed powder [1-4]. The composed material is usually cylindrically shaped in a press and fired later at high temperature (Fig. 1), to aggregate $\mathrm{ZnO}$ into grains. The aggregation process depends on how the used materials are mixed and squeezed. The mentioned additives play an important role during aggregation processes and are precisely selected by the varistor producer.

The aggregated grains form a structure which determines the final current-voltage characteristics of varistors. The well-prepared elements comprise relatively big grains that have the non-linear characteristics of junctions between them (Fig. 2a). Tiny grains (having ohmic contacts) are characterized by more linear characteristics (Fig. 2b). The ultimate varistor characteristics is the sum of characteristics of the junctions between the consecutive grains.

The voltage when varistor starts to conduct current is determined by the number of the grain junctions between the varistor electrodes. Thus, a shift in these voltage values is provided by changing the element thickness or applying a stack of the elements. The latter method is used for preparing varistors which protect high voltage power lines.

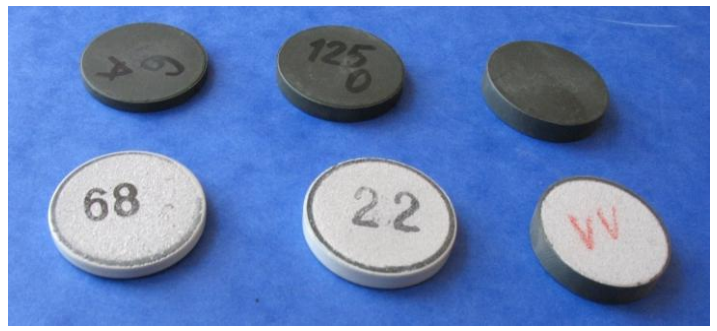

Fig. 1. Varistor samples: $\mathrm{ZnO}$ structures after being fired (top row) and with metalized contacts (bottom row); the thickness of the varistor structure determines the voltage value when the varistor begins to conduct current: left $-280 \mathrm{~V}$, middle $-440 \mathrm{~V}$, right $-660 \mathrm{~V}$.

a)

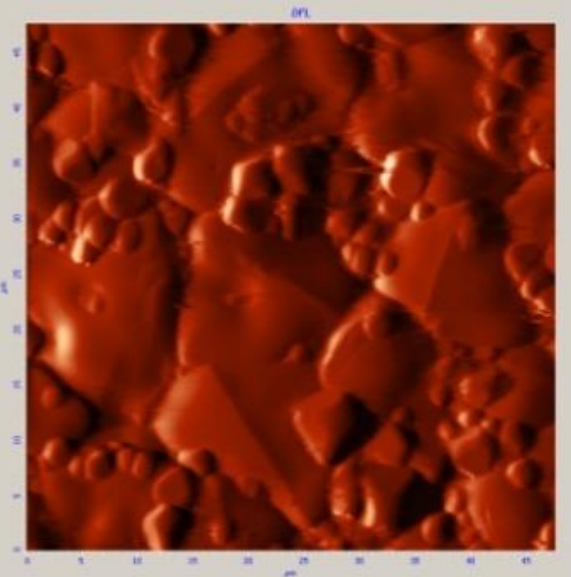

b)

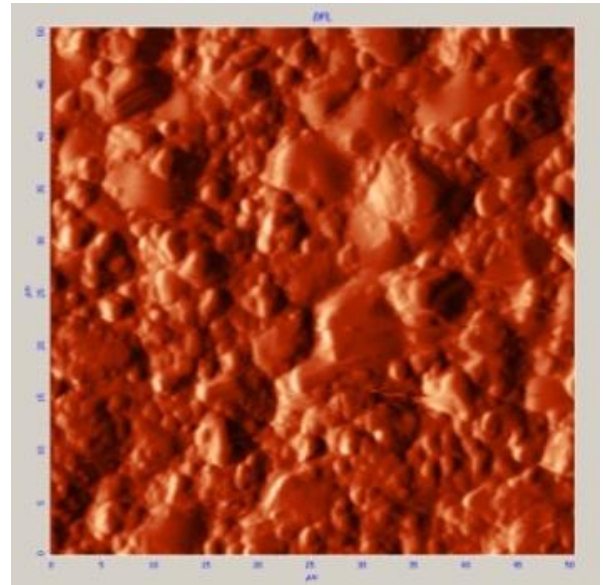

Fig. 2. Varistor structures in microscopic view (square area of size $50 \mu \mathrm{m} \times 50 \mu \mathrm{m}$ ): (a) well-prepared with large grains; (b) poor quality with smaller grains. 
It has to be underlined that the varistor structure is strongly influenced by working conditions. Particularly, a varistor can easily change its properties during an intense and even short current flow, when excessive overheating can reduce the nonlinearity of the grain junction characteristics or lead to forming conducting paths within the structure and result in a varistor failure.

The prepared $\mathrm{ZnO}$ cylinders have sprayed metal contacts and painted edges as isolating layers before being assembled into a plastic case (Fig. 3). Varistors are exposed to various environmental factors, like lightning or overvoltage spikes, together with excessive humidity during long-term exploitation (even $20 \div 30$ years old varistors are used without any maintenance in power systems). The mentioned factors influence their ageing processes that lead to cracks, conducting path formations or changes in grains which diminish the nonlinearity of varistor current-voltage characteristics [7, 8]. Especially, humidity invading the grained $\mathrm{ZnO}$ structure results in leakage current growth and diminish the varistors' ability to absorb intense current pulses because of excessive overheating which downgrades the nonlinearity of junctions between the grains.

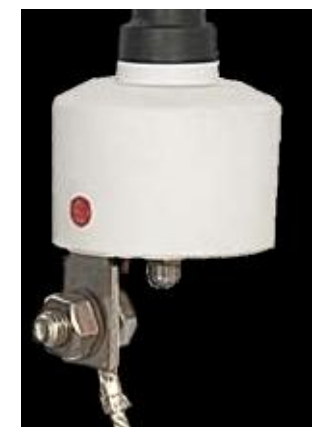

Fig. 3. Varistor packed in a plastic case for outdoor use as surge arrester.

\subsection{Electrical parameters}

Varistors are characterized by a strongly non-linear current-voltage characteristics (Fig. 4). This means that at a low voltage of a few hundred volts the leakage current of a varistor applied in a domestic power network should not exceed $1 \mathrm{~mA}$ (typically, such varistors can sustain the currents up to $10 \div 15 \mathrm{kA}$ during short $(\sim 10 \mu \mathrm{s})$ overvoltage pulses). When an overvoltage pulse occurred with a longer duration, e.g. $\sim 100 \mu \mathrm{s}$, then the sustained current is lower due to limited ability of heat dissipation within the $\mathrm{ZnO}$ structure.

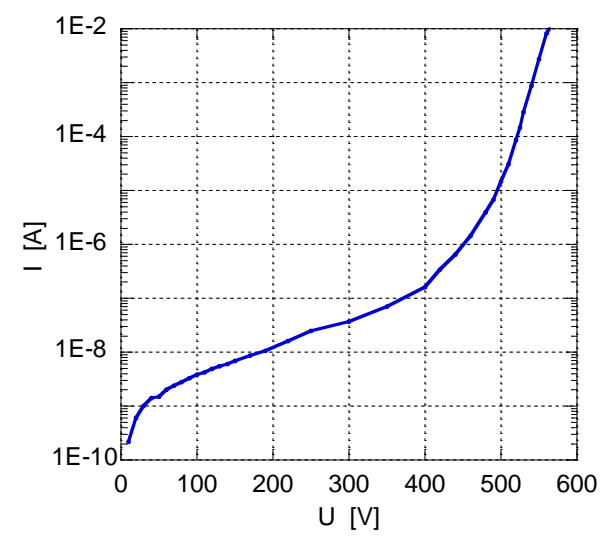

Fig. 4. Typical current-voltage characteristics of a varistor designated for working in a household power network. 
Varistors play their protective role by limiting overvoltage pulses in power supply lines. When the pulse is too excessive or lasts too long, the varistor will fail, cause a shortcut or excessive risk to the protected equipment [9]. The first mentioned case results in lack of further protection against overvoltages. The second one would trigger a power line protection fuse and cause an electricity cut off. Thus, varistor quality should be carefully controlled and monitored to avoid such cases. Moreover, the varistors having too intense leakage currents cause additional energy costs which can be now much higher than the costs of additional measurements.

\subsection{Ageing processes}

The induced stress caused by sequential overvoltages can form mini-cracks within varistors leading gradually to their complete degradation. Moreover, different working conditions during varistors exploitation cause various intensity of these ageing processes within their structures. The most important reasons of varistor ageing are overheating in the presence of overvoltage pulses or moisture penetration during changing weather conditions and leaks within their encasement. These conditions would increase the varistor current leakage and linearize their current-voltage characteristics.

The monitoring methods have to respond to various demands imposed by the power suppliers, when often only a yes/no decision system can meet the requirements of contemporary power industry. Such a system should memorize the intensity of all overvoltage pulses transmitted by the monitored varistor and estimate its ability to protect against such pulses in the future. Additionally, when the varistor shorts the protected power line, the failed device should be identified in a network by the monitoring system to help its easy replacement. The last remark is especially important when a disconnection due to a short circuit of a power line section contains a few varistors placed on high pylons in a hardly accessible area.

\section{Characterization of varistor quality}

The methods of varistor quality determination can be divided into two main groups. The methods from the first group can be performed when the varistors are polarized in their normal working conditions. The methods from the second group require additional, more stressed polarization (e.g., high voltage pulse injection). This paper will focus on the first group only due to possible applications in varistor quality monitoring systems in industrial power supply lines.

The simplest method of varistor quality determination is to measure its leakage current or to measure its nonlinearity by the third harmonic component [1]. The leakage current can be measured by applying a magnetic current sensor (e.g. a coil having a ferromagnetic or a steel core) and a current meter. The third harmonic can be measured by a specialized nonlinearity meter but it is more complicated than the leakage current measurements because a polarizing voltage in a power network has usually nonlinear components of unknown intensity. Therefore, in this case the system has to monitor nonlinearities of the power network voltage which polarizes the investigated varistor, too. The third harmonic component is a reliable factor of varistor quality and differs among the examined elements (Fig. 5). Table 1 presents data obtained for two groups of varistors: well prepared (marked with the letter "O") and having poor quality (marked with the letter "A"). For every "O" sample the third harmonic index value was higher. This indicates more nonlinear current-voltage characteristics.

Unfortunately, the third harmonic index does not give complete information about the ability to sustain overvoltage pulses when excessive overheating could lead to structural 
changes within the examined $\mathrm{ZnO}$ device. The thermal stability of the grained structure is determined by the balance of power loss of the protective element and the cooling capacity of the case which depends on outside temperature and humidity. The leakage current can be measured relatively easily but gives only general information about a varistor's quality. Its value preserves information about the history of varistor's use but has a limited ability of prediction how the varistor structure would sustain future overvoltage pulses. This is mainly determined by the ability to withstand extreme overheating which depends on numerous and changing factors. Therefore, the leakage current should be measured together with varistor temperature. It has to be underlined that the leakage current $I(t)$ comprises two components: the dominant imaginary part (shifted to the applied voltage $U(t)$ by $90^{\circ}$ ) and the real part (responsible for energy consumption). When the tested varistor is polarized by its working voltage, the measured leakage current is dominated by its imaginary part (Fig. 6). Moreover, the current $I(t)$ is so small that the sampled values need to be averaged to reduce efficiently noise and interferences.

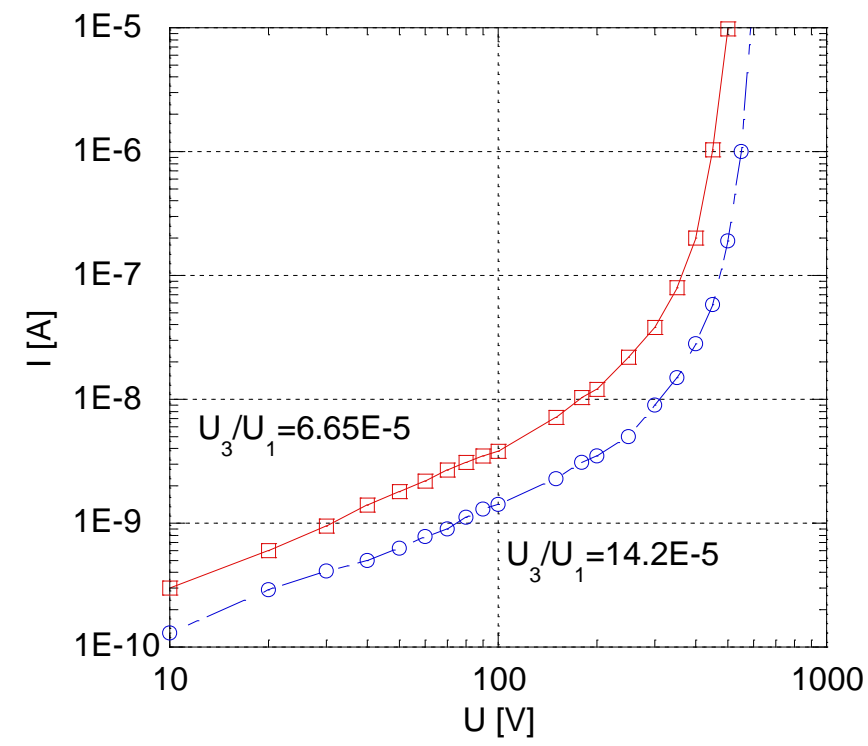

Fig. 5. Intensity of the third harmonic component $U_{3}$ related to current-voltage characteristics in varistors designated to work with nominal voltage of $280 \mathrm{~V}$; varistors were polarized during measurements by a sinusoidal voltage $U_{1}$ having a frequency of $10 \mathrm{kHz}$.

Table 1. Intensity of the third harmonic $U_{3}$ observed in some varistors designated to work with various nominal voltages; varistors were polarized during measurements by harmonic voltage $U_{1}=100 \mathrm{~V}$ at a frequency of $10 \mathrm{kHz}$.

\begin{tabular}{|c|c|c|}
\hline $\begin{array}{c}\text { Nominal } \\
\text { varistor } \\
\text { voltage [V] }\end{array}$ & Sample no. & $\begin{array}{c}U_{3} / U_{1} \cdot 10^{-5} ; \\
U_{1}=100 \mathrm{~V}\end{array}$ \\
\hline \hline 280 & O14 & 13.2 \\
\hline 280 & A98 & 5.9 \\
\hline 440 & O56 & 13.6 \\
\hline 440 & A79 & 6.95 \\
\hline 660 & O30 & 10.2 \\
\hline 660 & A29 & 6.65 \\
\hline 280 & O14 & 13.2 \\
\hline 280 & A98 & 5.9 \\
\hline
\end{tabular}


The above-mentioned problems of varistor quality monitoring in their working mode suggest that the most reliable method should base on measurements of their leakage current and temperatures at excessive overvoltage pulses. There is clear evidence that even not too intensive aging by repeated current pulses increases slowly varistor leakage currents (Fig. 6).

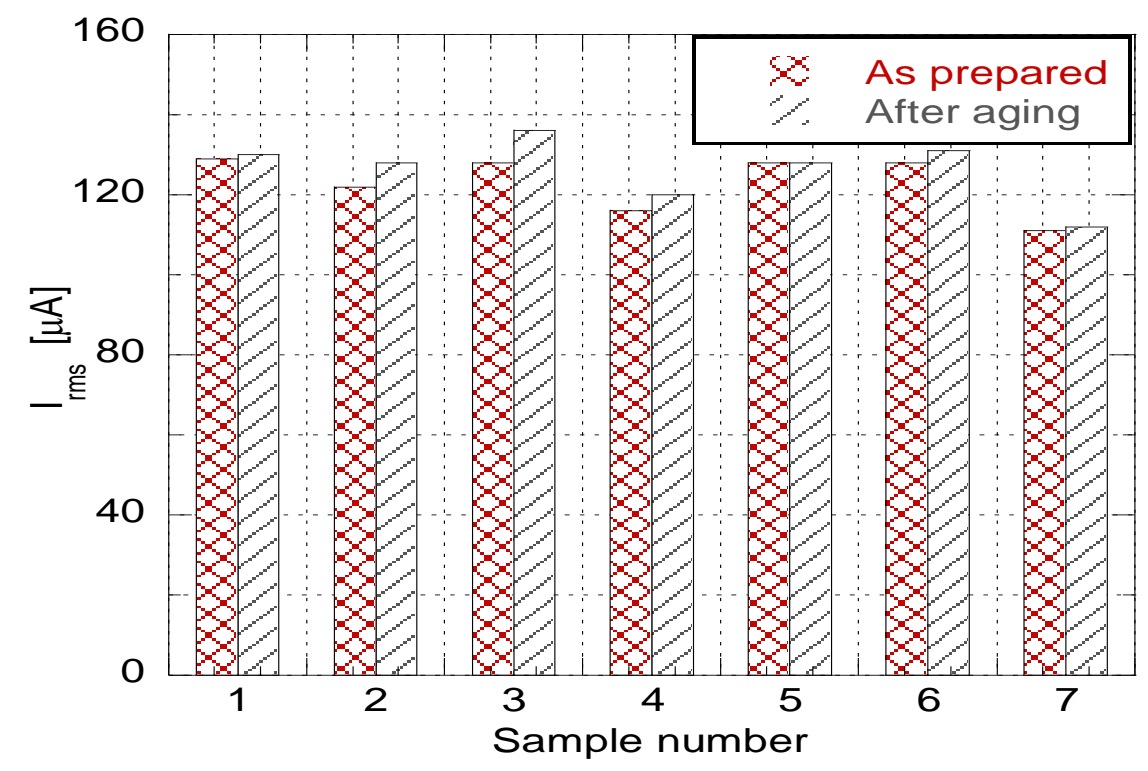

Fig. 6. Changes of the leakage current $I_{\text {rms }}$ in an exemplary set of seven varistors at a polarizing voltage of 350 Vrms before and after aging by applying 1000 current pulses of amplitude 0,5 kA and duration 8/20 $\mu$ s.

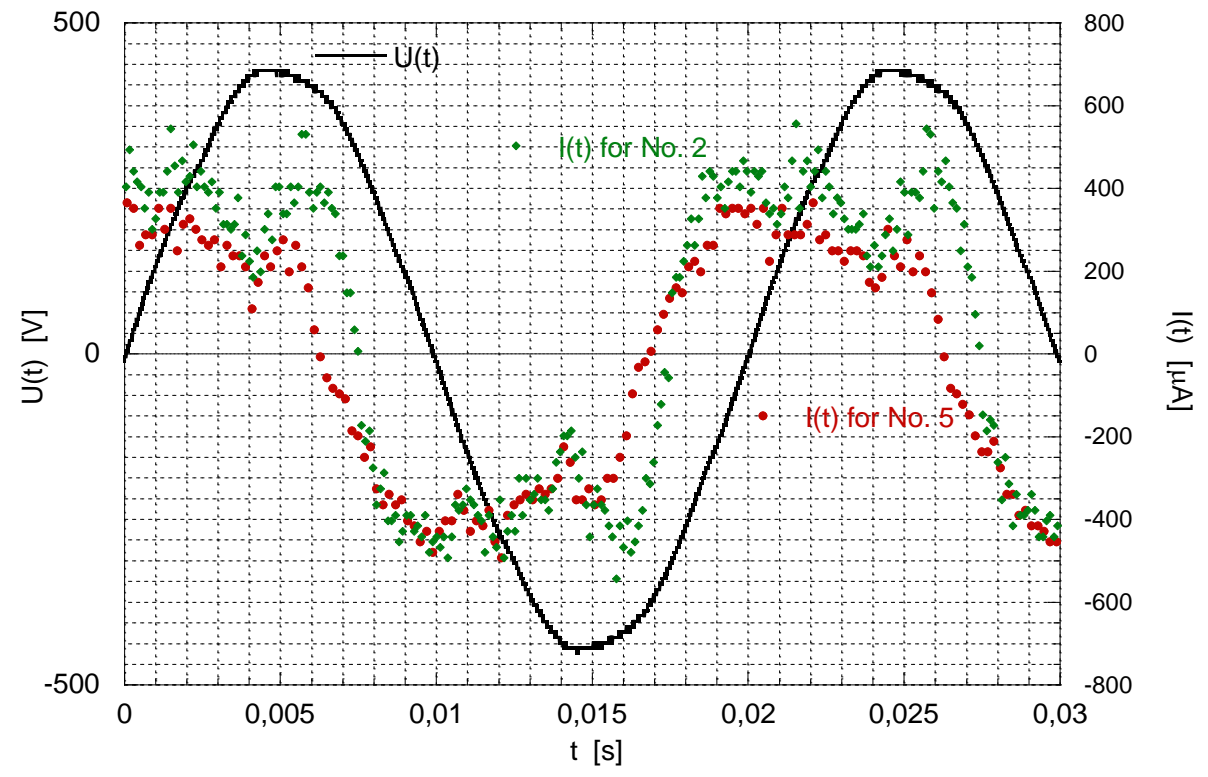

Fig. 7. Intensity of leakage current $I(t)$ flowing through varistors of poor (No. 2) and high (No. 5) quality polarized by sinusoidal voltage $U(t)$; the investigated varistor's working voltage $U_{\mathrm{RMS}}=275 \mathrm{~V}$; the current $I(t)$ was measured by current transformer, model Kyoritsu KEW 2432.

The leakage current can be easily measured during overvoltage pulses by a cheap electronic unit, isolated from the power supply line circuit (Fig. 7). The more problematic are varistor temperature measurements because the temperature sensor has to be powered and placed close to the varistor structure. Otherwise, such placement increases the probability of moisture invasion into the grained structure. 
It should be underlined that the varistors are often mounted on tall pylons and therefore are subject to changing weather conditions. Thus, a monitoring system which could be accepted by the industrial customers has to meet a few contradictive requirements: low price, yes/no decision about varistors quality at high rate of their failure detection, durable operation without any maintenance in harsh weather conditions. Furthermore, it would be desirable to propose a method which would allow more advanced measurements as well as to meet various market requirements.

\section{Measurement system for varistor quality monitoring}

Various systems of varistor quality monitoring have been described and proposed for laboratory and industry applications [4, 8, 10-13]. The most commonly recommended are systems which are cheap and do not require additional varistor polarization. Such systems monitor events of excessive varistor temperature or current during lightning (Fig. 8) and have limited applications for the voltage of power networks higher than $230 \mathrm{~V}$ because of the inability of reading the varistor state by the electromagnetically supplied RFID transceiver which works at short distances only [10]. The system neglects the influence of numerous excessive overvoltage pulses that are lower than the limit established by the producers but can influence varistor degradation. The presented solution can be applied only when the varistors are placed in stable conditions with a very limited influence of moisture. Then, monitoring of overheating or excessive currents by cheap fuses could be considered as a sufficiently reliable factor of varistor quality. Another proposed solution requires measurements of varistor leakage current only, which can be easily performed in low voltage power networks by mounting electrical coils in the varistor circuit [10]. The method gives general information about varistor quality only and can be misleading because it does not take into account changes of varistor temperature and surrounding humidity even in a laboratory environment (mainly due to seasonal heating). Thus, the additional temperature measurements can determine more precisely conditions of varistors overheating in the presence of overvoltage pulses.

Even a more convincing example of the necessity of temperature measurement is the case of varistors exposed to changeable weather conditions, especially to a wet environment which reduces varistor impedance and can result in overheating of its structure. Therefore, a new method has been proposed. It requires measurements of varistor current and temperature during numerous overvoltage pulses (Fig. 9). The proposed solution is a new one because the measurement unit can be functional during a few years without any maintenance. It is worth mentioning that there are electric pylon-mounted varistors that can work even tens of years there. Therefore, only long-term working test units can be accepted by industrial customers.

The unit requires extremely low power because it remains in the standby mode mainly. The exception is when it is waked by a reset signal induced when an overvoltage pulse occurs (Fig. 9). Then, the microcontroller measures the intensity of the occurred overvoltage pulse by counting the time when the signal at the output of the AC/DC converter is greater than the assumed threshold level. The proposed solution means that we can apply the microcontroller which needs in the standby mode a power consumption not exceeding a few $\mu \mathrm{W}$ and less than $1 \mathrm{~mW}$ when the output voltage of the AC/DC converter is measured [14]. Such a small energy can be delivered by a lithium battery which can be used in the system designed to last ten or even more years. To increase the reliability of the system, an alternative power harvesting unit (e.g., solar power, thermal energy) can be attached as well. 


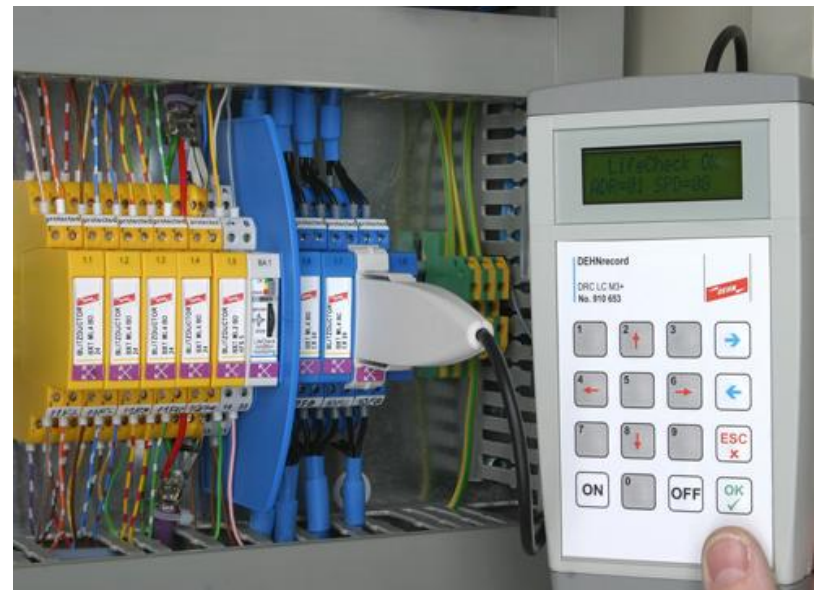

Fig. 8. Measurement system of varistor quality in a household power network proposed by DEHN [10].

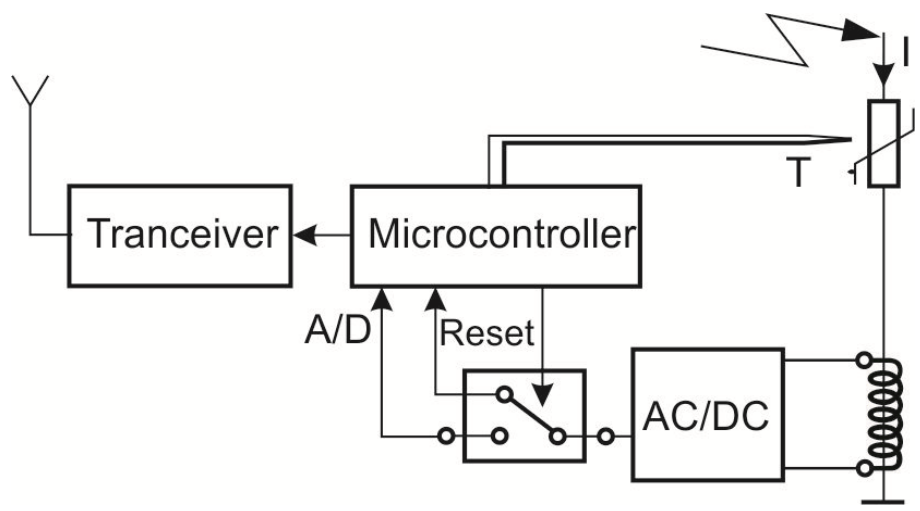

Fig. 9. Measurement system for varistor quality monitoring.

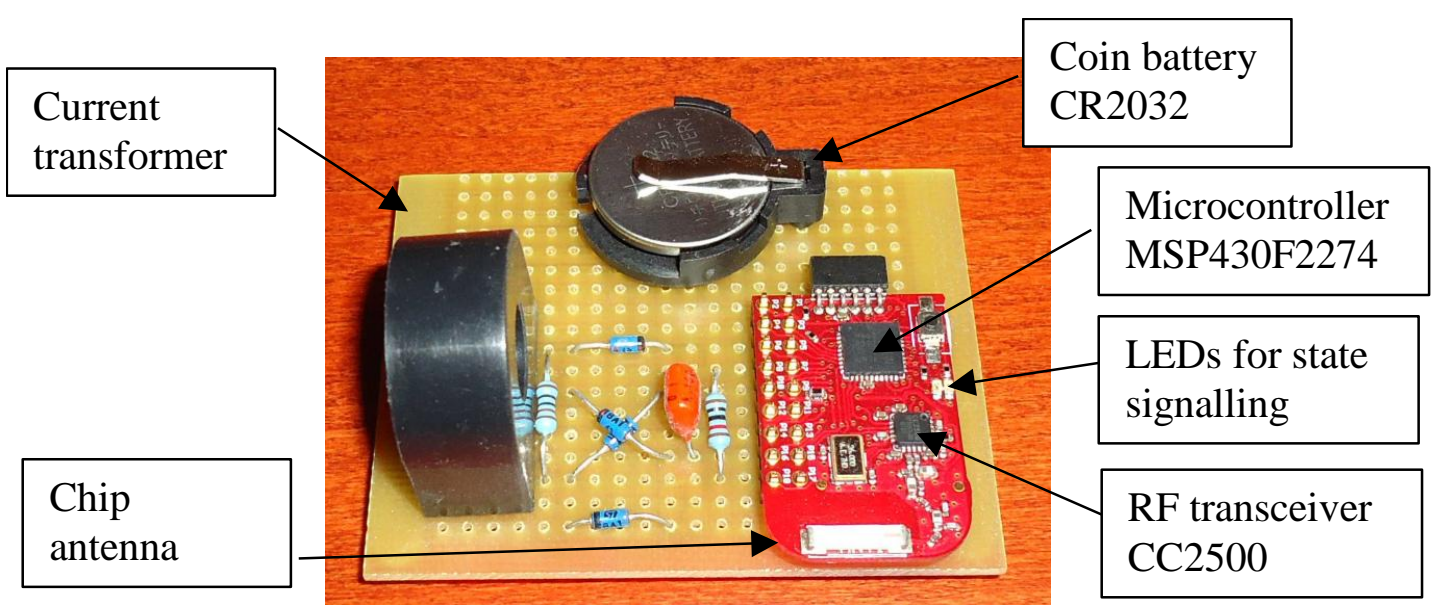

Fig. 10. The prototype version of the proposed measurement system with the RF transceiver for wireless communication.

The same microcontroller can measure temperature by an additional sensor. A more advanced solution can apply the newly developed SAW-based radio temperature sensor [15]. 
This means that the temperature sensor can be attached directly to the varistor case without wires but at the cost of an additional transceiver unit connected to the microcontroller.

The developed prototype version of the measurement system (Fig. 10) comprises a current transformer with a diode bridge and an RC filter which generates the reset signal to the microcontroller and integrates the overvoltage signal to measure its intensity. The current consumed by the working circuit depends on the functionality of the system. When the wireless communication module is switched off, the supply current is about 2,4 $\mathrm{mA}$ in the activated state (during voltage measurement by the built-in $\mathrm{AD}$ converter and saving data in the EEPROM on-chip memory) and drops to $1,4 \mu \mathrm{A}$ in the standby mode. Such current consumption is higher than the values suggested by the chip suppliers [16]. Despite the observed discrepancy, the applied battery, type CR2032 of $210 \div 260$ mAh capacity, should maintain the system working even for ten years.

Results of the consecutive current measurements induced by overvoltages can be memorized using a few digits only to compare with the established threshold criterion to give a clear alternative answer about varistor quality (e.g. yes/no answer or a percentage of the varistor wear). Moreover, the same system can measure the leakage current and transmit the data by a transceiver. When more frequent measurements have to be performed, the system can be powered by an independent energy source, like a small and cheap solar cell. The described system, which is at present in further development, can be also used to detect and inform when the varistor caused a shortcircuit, which helps the power network service teams to repair the line.

\section{Conclusions}

Varistors made of $\mathrm{ZnO}$ grains were characterized and their electrical properties were presented. Some selected problems of varistor quality monitoring have been considered as well. The aging processes due to overheating and moisture ingress that lead to varistor degradation have been also taken into account. These processes require continuous check-ups of varistors ability to protect the power lines against destruction induced by overvoltage pulses. Some newly proposed methods of their quality monitoring during exploitation were enclosed. The measurement systems which are presently applied for this aim were discussed by underlining their advantages and drawbacks for use in industrial applications. A new proposal was enclosed and the main advantages of the patented circuit were underlined. The presented measurement unit can sustain effective varistor quality monitoring without necessity of any maintenance within a period of ten years at least. Additionally, the proposed unit can identify the failed varistor even when the power line is cut off. A very similar measurement unit can be used for varistor leakage current measurements. Therefore, the proposed solution is a comprehensive device which should meet various requirements of industrial applications. Moreover, the proposed unit can be developed into a monitoring network, used in an area of high-risk lightning because of economical reasons.

\section{Acknowledgements}

This research was financed by the Polish Ministry of Science, project No. 3043/B/T02/2010/38, 2010-2011.

\section{References}

[1] Jaworski, M. (1999). Endurance prediction of metal-oxide $\mathrm{ZnO}$ varistors from current production, $P h . D$. Thesis. Wroclaw University of Technology. (in Polish) 
[2] Sedlak, P., Tofel, P., Sedlakova, V., Majzner, J., Sikula, J., Hasse, L. (2011). Ultrasonic spectroscopy of silicon single crystal. Metrol. Meas. Syst., 18(4), 621-630.

[3] Lira, J., Macedo, E., Costa, E., Freire, R., Luciano, B. (2007). ZnO surge arresters diagnosis using microcontroller. In Instr. \& Meas. Technology Conference - IMTC 2007, Warsaw, Poland, 1-6.

[4] Christodoulou, C.A., Avgerinos, M.V., Ekonomou, L., Gonos, I.F., Stathopulos, I.A. (2009). Measurement of the resistive leakage current in surge arresters under artificial rain test and impulse voltage subjection. IET Science, Measurement and Technology, 3(3), 256-262.

[5] Abdul-Malek, Z., Noorden, Z.A., Yusoff, N. (2010). The interpretation of return voltage curve of ZnO varistor based on a new diagnostic parameter. In UPEC2010.

[6] Sikula, J., Schauer, P., Vasina, P., Sikulova, M., Koktavy, B., Chobola, Z., Navarova, H., Pazdera, L. (1994). 1/f Noise in metallic thin films. In AIP Conf. Proc. 371, 59-64, doi: http://dx.doi.org/ $10.1063 / 1.50886$.

[7] Furgał, J. (2009). Analysis of voltage-current dependencies of metal oxide surge arresters in different exploitation conditions. Electrotechnical Review, 85(6), 25-28.

[8] Heinrich, C., Hinrichsen, V. (2001). Diagnostics and monitoring of metal-oxide surge arresters in highvoltage networks-comparison of existing and newly developed procedures. IEEE Transactions on Power Delivery, 16(1), 138-143.

[9] Furgal, J. (2009). Analysis of overvoltages in windings of power transformers protected by use of metal oxide surge arresters. European T. on Electrical Power, 19(3), 400-410.

[10] LifeCheck® SPD Test Device DRC LC M3+, (2011), DEHN + SÖHNE GmbH + Co. Neumarkt, Germany, www.dehn.de. (2012).

[11] Smulko, J., Hasse, L., Kaczmarek, L. (2011). Method and system for varistor degradation assessment. Polish patent disclosure, No. P.396244. (in Polish).

[12] Abdul-Malek, Z., Aulia, N. (2008). Portable device to extract resistive component of the metal oxide surge arrester leakage current. In Australasian Univ. Power Eng. Conf. (AUPEC'08), P245, 1-5.

[13] Schillert, H., Schubert, M., Steinfeld, K. (2008). Device for monitoring the leakage current of a surge arrester. US patent No. 7336193B2.

[14] Texas Instruments mixed signal microcontroller, http://www.ti.com/lit/ds/slas504f/slas504f.pdf. (2012).

[15] Scholl, G., Korden, C., Riha, E., Ruppel, C.C.W., Wolff, U., Riha, G., Reindl, L., Weigel, R. (2003). SAW-based radio sensor systems for short-range applications. IEEE Microwave Magazine, 4(4), 68-76.

[16] Atmel, ATtiny13, ATtiny13V, 8-bit Microcontroller with 1K Bytes In-System Programmable Flash, Rev. 2535H-AVR-10/07. (2011). 